\title{
Cryostat for Testing RF Power Couplers
}

\author{
M. Kuchnir et al. \\ Fermi National Accelerator Laboratory \\ P.O. Box 500, Batavia, Illinois 60510
}

March 1996

Submitted to the 1995 Cryogenic Engineering Conference (CEC), Columbus, Ohio, July 17-20, 1995. 


\section{Disclaimer}

This report was prepared as an account of work sponsored by an agency of the United States Government. Neither the United States Government nor any agency thereof, nor any of their employees, makes any warranty, express or implied, or assumes any legal liability or responsibility for the accuracy, completeness, or usefulness of any information, apparatus, product, or process disclosed, or represents that its use would not infringe privately owned rights. Reference herein to any specific commercial product, process, or service by trade name, trademark, manufacturer, or otherwise, does not necessarily constitute or imply its endorsement, recommendation, or favoring by the United States Government or any agency thereof. The views and opinions of authors expressed herein do not necessarily state or reflect those of the United States Government or any agency thereof. 


\title{
CRYOSTAT FOR TESTING RF POWER COUPLERS
}

\author{
M. Kuchnir, M.S. Champion, K.P. Koepke and J.R. Misek \\ Fermi National Accelerator Laboratory* \\ Batavia, IL, 60510, USA
}

\begin{abstract}
Similar to the power leads of accelerator superconducting magnets, the power couplers of accelerator superconducting cavities are components that link room temperature to superfluid helium temperature for the purpose of energy transfer. Instead of conducting kiloamperes of current they guide megawatts of RF power between those two temperatures. In this paper we describe a cryostat designed for testing the performance of these components and measuring their heat loads. A special feature of this cryostat is its minimum liquid inventory that considerably simplifies safety related requirements. This cryostat is part of a Fermilab facility contributing to the international collaboration working on TESLA (TeV Electron Superconducting Linear Accelerator). This facility is now operational and we will be presenting specifications as well as performance data on the cryostat as well as the first pair of power couplers tested with it.
\end{abstract}

\section{INTRODUCTION}

One of the TESLA (TeV Electron Superconducting Linear Accelerator) ${ }^{1}$ essential components is the Input RF Power Coupler that transmit the $1.3 \mathrm{GHz}$ Power from room temperature to the $1.8 \mathrm{~K}$ environment of the superconducting accelerating cavities. These articulated coaxial transmission lines have a wave-guide to coax transition at room temperature, a ceramic window at $78 \mathrm{~K}$, and an outer conductor extremity connected to the superconducting beam-tube between the cavities.

In order to test the power handling capability of these power couplers and measure their heat loads on the refrigeration system we designed the cryostat described here. It measures their heat loads by thermal sinking them through heatmeters.$^{2}$ A resonant ring containing a single cell superconducting cavity between two power couplers is used with the available $100 \mathrm{~kW}$ power source in order to test them RFwise up to the $1 \mathrm{MW}$ power level required for HPP (High Power Processing) ${ }^{3}$ of the TESLA cavities in situ. This arrangement is schematically shown in figure 1 . The cavity together with the colder segments of the couplers are attachable to the cryostat as an evacuated unit. A special container for shipment of this unit was also built. This allows for the chemical surface treatment of the cavity and clean room assembly of this three components unit to be performed at already existing facilities of participants in the TESLA collaboration.

In this paper we describe the cryostat, its operation and one of the first tests using it. This test verified the cryostat operation and measured the static heat load of the two

Fermi National Accelerator Laboratory is operated by Universities Research Association, Inc. under contract with the U.S. Department of Energy. 


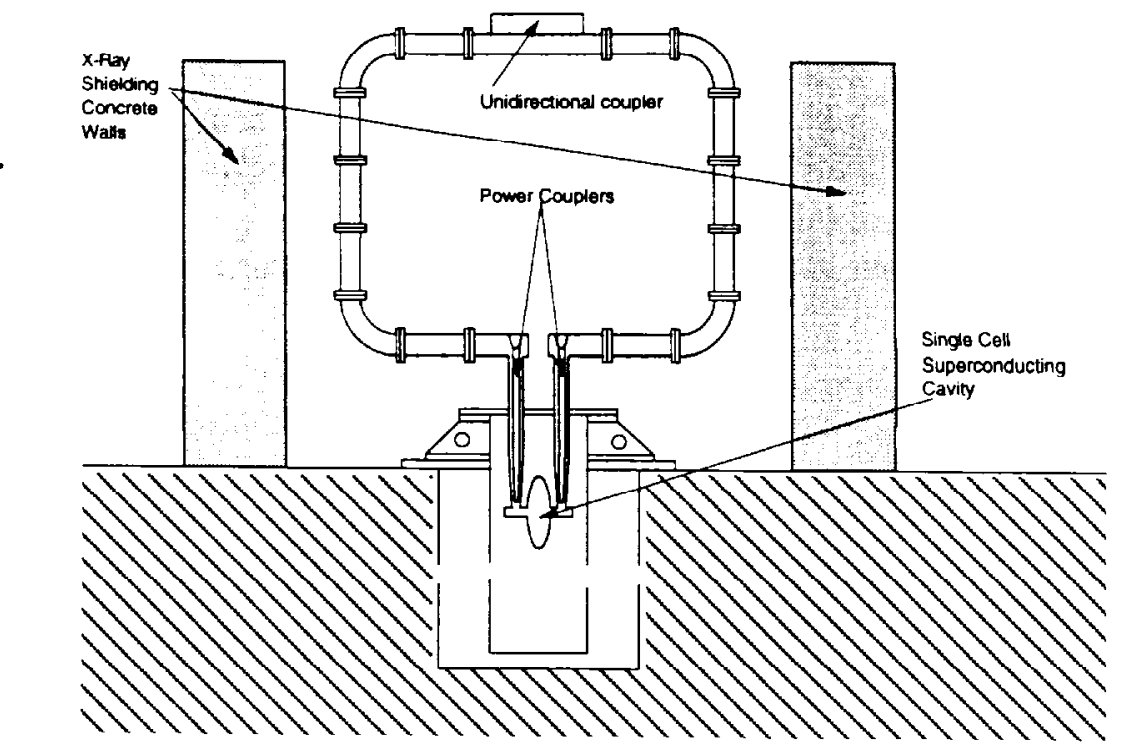

Figure 1. Resonant ring testing concept

power couplers in use. Performance under RF power was precluded by the lack of the required chemical treatment of the superconducting cavity.

\section{CRYOSTAT DESCRIPTION}

The length of the cryostat is dictated by the power couplers length. As a consequence it is rather short $(0.7 \mathrm{~m})$, also the separation between the couplers is dictated by simulation to the TESLA cavity cell-coupler distance resulting in a axis to axis distance of $(0.203 \mathrm{~m})$. This cryostat fits in an existing sunken dewar of $0.44 \mathrm{~m}$ internal diameter that is used here just as a vacuum vessel.

In order to make the cryostat inherently safe and avoid need for boiler code certifications etc. the liquid He inventory is minimized to 1.6 liters and the operation is based on continuously flowing cryogens from a liquid $\mathrm{N}_{2}$ trailer and a liquid $\mathrm{He} 500$ liter storage dewar.

The cryostat is formed by a stainless steel top plate, a copper $\mathbf{L N}_{2}$ plate with its aluminum shield that surrounds a copper LHe plate and its aluminum shield that in tum surrounds the pure niobium piece. The surrounding is not complete since race track shaped holes in the copper plates are needed for the couplers, plumbing lines and instrumentation cables. The gaps remaining in these holes are closed for infrared radiation purposes with closer fitting multilayered insulation mats. Four G11CR fiberglass-epoxy rods form the structural frame that holds together the plates and the cradle holding the niobium piece. Figure 2 presents these components in a simplified schematic. The simplification required for figure 2 substitutes by a single stainless steel bellows the rather sophisticated mounting system of the power coupler to the top plate. This mounting system allows the motions required for RF tuning of the coupler to the TESLA cavities as well as the resonant ring in this test arrangement besides taking care of thermal expansion differentials.

The stainless steel top plate is $25.4 \mathrm{~mm}$ thick. Besides the circle of bolts that seals it to the dewar and the two flanges for the coupler adapters it has 10 penetrations and 4 blind screw holes for the set of G11CR fiberglass-epoxy rods. Pairs of these penetrations are for the LHe transfer lines, $\mathrm{LN}_{2}$ lines, rotary feedthroughs, electrical wires and fiber optic feedthroughs, vacuum and pump out ports. The penetrations for LHe transfer lines are equipped with Cajon fittings for $12.7 \mathrm{~mm}$ OD transfer lines. These rigid $U$ shaped transferlines have $6.35 \mathrm{~mm} \mathrm{ID}$ and are used for both the incoming liquid and the exiting cold gas from the copper plate and cryopump (Exhaust).

The penetrations for the $\mathrm{LN}_{2}$ lines and the LHe Vent line are double walled elbows using the insulating vacuum to prevent their cold inner tube $(6.35 \mathrm{~mm}$ diameter $)$ from 


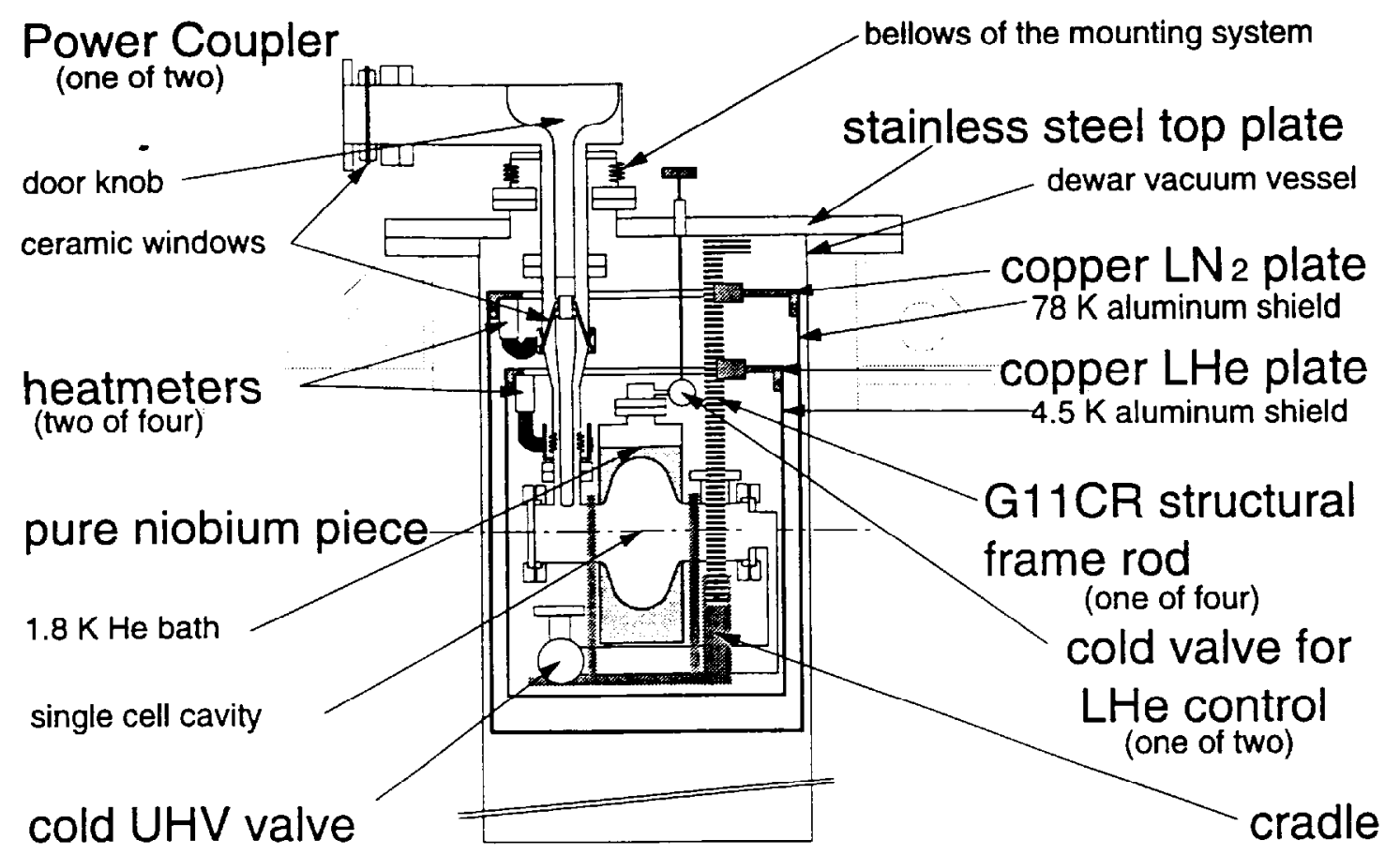

Figure 2. General mechanical structure of the cryostat

cooling the plate. Since thick frosted coating on these lines was not a concern, "Hytron" thermoplastic hoses (manufactured by Imperial Eastman) have been used here with good results although these temperatures are well below their specifications . The rotary "Ferrofluidic" feedtroughs are used to permit the control of the two cold valves through $6.35 \mathrm{~mm}$ diameter G11CR rods that extend their stems inside the cryostat.

The copper plates are $9.53 \mathrm{~mm}$ thick discs of OFHC copper to which are brazed two copper-magnet-bus-hollow conductors and two small OFHC copper discs. Through the hollow conductors will flow the cryogens that determine the temperature of the plates. One of these is $2.16 \mathrm{~m}$ long and has $5.79 \times 5.79 \mathrm{~mm}$ cross section with a $3.18 \mathrm{~mm}$ diameter hole, the other has a $14.3 \times 25.4 \mathrm{~mm}$ cross section with a $6.35 \mathrm{~mm}$ diameter hole and form the rim of the plate. The outside of this rim is then machined at a $2.0^{\circ}$ angle to form a conical surface for thermal contact with its aluminum shield. The two small discs add thickness to the plates locally for the 1/2-14NPT pipe threaded hole used for the attachment of the heatmeters

The $78 \mathrm{~K}$ and $4.5 \mathrm{~K}$ shields are made from $3.18 \mathrm{~mm}$ thick aluminum plate rolled and welded to form flat based cylindrical cans. The rims are internally machined at a $2.0^{\circ}$ angle to closely contact the conic surfaces of the respective plate rims. Three race-track shaped holes equally spaced around are machined partially intersecting the conic surface to permit the introduction of a tool for the uncoupling of this binding conic contact. These holes are also used by special fasteners to prevent gravitational induced de coupling. The conic surfaces are lightly coated with Apiezon L grease to improve thermal contact and ease of disassembly. The thermal contact of these conic surfaces, helped by the copper/aluminum differential thermal expansion coefficient proved to be completely satisfactory.

The pure niobium piece was formed at the Laboratory for Nuclear Studies of Cornell University and further modified by e-beam welding to it the LHe reservoir, the pulled-out tubes and flanges. These latter operations were done at Fermilab. Noteworthy are the seals to the stainless steel mating flanges that use Helicoflex "O-rings". In particular, one of them seals the superfluid He containing reservoir from the insulating vacuum. This piece together with the Ultra High Vacuum (UHV) manifold and its all metal valve are held to a cradle that allowsus to position it easily on a table or attach it and the lower parts of its two couplers to the cryostat frame or to a shipping frame. So the niobium piece consists of a beam tube with two radial pullouts (for the couplers) between which it expands in an 
elliptical single cell cavity. This cavity is coaxially surrounded by a cylindrical liquid He container also with a radial pull-out which is parallel and in between the other two. The extremities of the beam tube and pull-outs have shoulders that fit into special stainless flanges designed to seal with Helicoflex metal "O-rings". The entire piece is made exclusively of high purity niobium stock and electron-beam welded together. The niobium piece, its cradle and the ceramic-window-sealed colder halves of the two couplers, plus a manifold ending in an all metal valve, V1, are detachable as one UHV system that can be shipped for chemical polishing and assembly in a clean room.

The four supports that form the structural frame of the cryostat were machined out of a G11CR plate. They have the shape of an inverted long and narrow $L$. The foot of each $L$ is attached to the bottom of the stainless top plate by means of a 1/4-20 screw into a threaded hole $12.7 \mathrm{~mm}$ deep. The long vertical legs have a $12.7 \times 25.4 \mathrm{~mm}$ cross section. Horizontal threaded holes are used to hold the copper plates and heat-sink the supports at the copper plates temperatures. This is accomplished by means of copper coated screws and copper coated stainless adapters. The LN2 cooled copper plate is at $76.2 \mathrm{~mm}$ below the stainless top plate and the LHe cooled copper plate is 102 . $\mathrm{mm}$ further down. The horizontal holes used for the attachment of the cradle and its pure niobium piece (at $1.8 \mathrm{~K}$ ) are 241 . mm further down.

The four heatmeters are very similar. The two, designed for the $80 \mathrm{~K}$ region, have a $2.1 \mathrm{~mm}$ long stainless steel body segment between copper segments containing $100 \Omega \mathrm{Pt}$ RTDs (Resistance vs. Temperature Devices). The other two, designed for the $5 \mathrm{~K}$ region, have a $0.5 \mathrm{~mm}$ long stainless steel body segment between copper segments containing $100 \Omega, 1 / 8$ w Allen Bradley carbon composition RTDs.

Each heatmeter has a $45 \Omega$ calibration heater and an aluminum thermal shield. The contact to the heat sinking plates is by means of 1/2-14 NPT pipe threads (copper to copper) and to the couplers through copper braided cables and copper clamps. Copper powder loaded grease is used in these contacts and care is taken to prevent the braided cables from touching anything.

Not shown in figure 2 is the cavity ultra high vacuum pumping line, it is composed of three sections. The section from $300 \mathrm{~K}$ to $80 \mathrm{~K}$ is formed by a zigzag of three coaxial vertical tubes in order to reduce heat conduction. The middle section is a $31.75 \mathrm{~mm}$ diameter $0.11 \mathrm{~m}$ long horizontal stainless tube joining the other (vertical) sections through mitered welds. The lowest section has a brazed copper sleeve $.32 \mathrm{~m}$ long around it. This sleeve has a cooling channel that makes it act as a cryopump. This line terminates with Conflat 2.75 flanges. The flange at room temperature is after an elbow adapted to the top plate and the flange at low temperature connects to the all metal valve of the detachable UHV system.

The $1.8 \mathrm{~K}$ bath pumping line, also not shown in figure 2, starts as a $6.35 \mathrm{~mm}$ diameter stainless tube leaving radially from the cap of the bath and connecting to the shell side of the heat exchanger. The shell of this heat exchanger is made out a $15.9 \mathrm{~mm}$

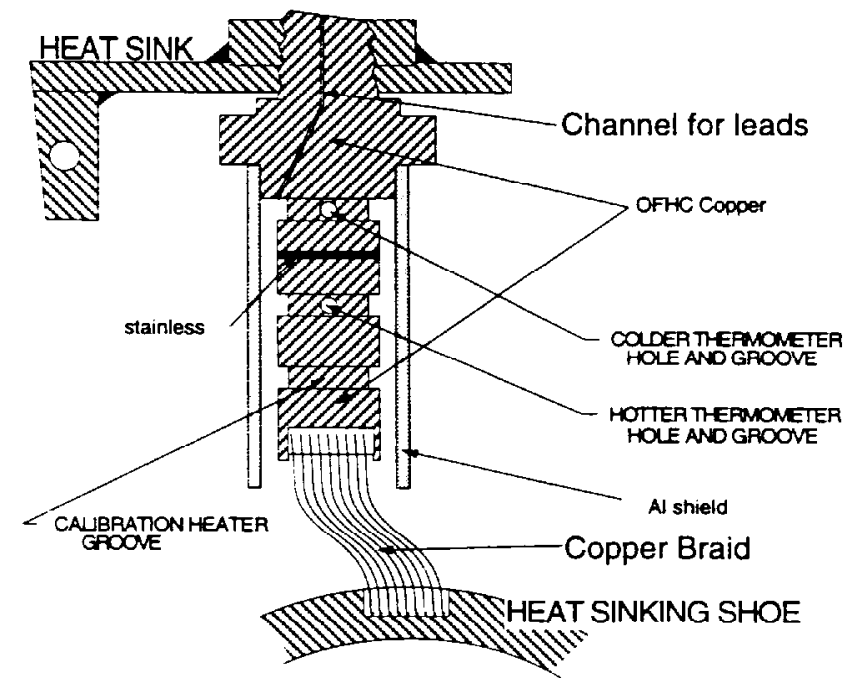

Figure 3. Heatmeter mechanical schematic. 
diameter, $.89 \mathrm{~mm}$ wall, $0.53 \mathrm{~m}$ long stainless tube curved in a semi-circle. This semicircle lays below and along the inner edge of the copper LHe plate without touching it. Inside the heat exchanger, seven stainless capillary tubes each $0.79 \mathrm{~mm} O \mathrm{OD}$ $0.15 \mathrm{~mm}$ wall $\times 1.70 \mathrm{~m}$ long are connected in parallel and coiled. They carry and cool the liquid helium to the JT valve. After the heat exchanger this line continues as a $19.0 \mathrm{~mm}$ OD stainless tube to a bayonet type port on the top plate. Care was taken to insure that this line slopes up all the way to prevent formation of liquid pools above the bath.

The cap of the $1.8 \mathrm{~K}$ bath is a rather busy component that contains an electrical feedthrough with 9 wires, the pumpout tube, the refill tube, the cooldown tube and the supports for three electrical connectors one inside and two outside besides the shoulder for the sealing flanges. The feedthrough is made from Stycast 2850 in a well tested style 4 . One of the outside connectors is of SMA type and is used for the twisted pair leads of the capacitive liquid Helium level sensor. The other two are commercial 9 pin connectors based on diallyl phthalate. So, before sealing the cap to the bath, a cable with the 4 leads from a RTD, 2 leads from the level sensor and two leads from the immersed bottom heater is plugged in the inner connector. Also, before sealing the cap to the bath, a Teflon tube gets connected to the cooldown tube so that the cold helium is directed to the bottom of the bath. Therefore, the end of the cooldown tube inside the cap points directly down ready for connection to the Teflon tube that will extend it to the bottom of the bath. The refill tube however brings the expanding cold mixture from the JT valve pointing it horizontal and tangential to the inner wall for a phase separation action and minimum disturbance of the accumulated liquid. The pumpout tube leaves the cap from the cylindrical side wall radially.

The capacitive liquid helium level sensor is made out of 3 stainless steel strips (.13 mm thick, $12.7 \mathrm{~mm}$ wide shims 260 . mm long) separated with $1.59 \mathrm{~mm} \times 9.52 \mathrm{~mm}$ spacers, $25.4 \mathrm{~mm}$ apart, each made out of 7 layers of adhesive Teflon tape buildup to a thickness of $0.62 \mathrm{~mm}$. The outer strips form the external grounded plate of the capacitor. This flexible probe is inserted in the bath space along the external equator of the single cell cavity. Therefore its response to the level is non-linear in a calculable way. The circuit used has been described by S.J. Collocott ${ }^{5}$. In this system we placed the front end frequency to voltage converter (an integrated circuit) on the connector inside the cryostat to reduce the parasitic capacitance and reduce noise pick up.

\section{MODES OF OPERATION AND FLOW DIAGRAM}

The cryostat is cooled and run by continuously flowing liquid He from a 500 liter storage dewar and liquid $\mathrm{N}_{2}$ from an existing storage trailer. Figure 4 is the flow diagram of the system showing these components and the cryostat plumbing. Non conventional schematic symbols and lines are used to give a more pictorial representation of the actual system. Valve V1 is an all metal valve which is operated only when the system is at room temperature and is kept open when the system is in the dewar for cooling or running. It is not part of the helium flow system. The liquid from the storage dewar and the part of it that is vaporized by the transfer tube heat leak are handled differently according to the operational mode.

After establishing the plumbing connections, vacuum leak testing the different volumes, leaving them with the appropriate gas and valve settings the cool down starts by flowing nitrogen from the storage trailer through the $\mathrm{LN}_{2}$.copper plate. In the first stage of the cool down mode, the vent plumbing, valves $\mathrm{V} 3$ and $\mathrm{V} 2$ are closed directing the cold helium to cool the lower section of UHV pumpline (cryopump) and the copper LHe plate exiting through the exhaust exit plumbing. In the second stage, once the UHV cryopump is cold, valve V3 is open directing the helium to further cool the copper LHe plate and the pure niobium piece leaving the system via the bath pumpout line. This stage should be paced by the conduction cooling of the heavy end-flanges of the cavity.

In the operating mode, valve V3 is closed and the incoming mixture reaches the phase separator where the gas is directed to the now open vent exit plumbing while the liquid continues to be used to cool the cryopump section of the UHV pumping line and the copper LHe plate leaving the cryostat via the exhaust exit plumbing. Part of this flow is diverted to the $1.8 \mathrm{~K}$ bath via the cold heat exchanger and the $\mathrm{V} 2$ valve which here acts as a the Joule Thompson expansion valve (JT). The aperture of this valve is adjusted to maintain 
the level in the bath stable above the cell wall. The time constant characteristic of this control is suitable for manual control, but future upgrade includes an automatic control for this function.

In figure 4, the heat exchanging parts of the flow lines are indicated with their diameter and length in inches. The connections are made with $6.35 \mathrm{~mm}$ OD $\times 0.89 \mathrm{~mm}$ wall stainless tubes welded or brazed. The heat exchanger before the V2 valve has been described above as part of the $1.8 \mathrm{~K}$ bath pumping line. The phase separator has a volume of less than $50 \mathrm{~cm}^{3}$ and is probably not optimal. The vent tube coming out of it is being redesigned to go trough a serpentine cooling line in the LHe shield prior to exiting the cryostat.

The general control of the operation is done by the pressure in the LHe storage dewar and the flow limiting rotameter valves downstream in the exhaust and vent exit plumbing. These helium flows determine the $4.5 \mathrm{~K}$ shield temperature and enable the JT valve (V2) effective operation, which together with the pumping on the helium bath surrounding the cavity and the heat load determines the cavity temperature.

Besides the thermometers and heaters in the heatmeters that have been described above and the sensors in the helium bath there are RTDs installed in the shields and plates. Sixteen RTDs are multiplexed into four hardware channels. Other instruments installed are four pressure gauges, two flow meters, and two liquid level indicators.

The data acquisition system is based on hardware and software designed and built at

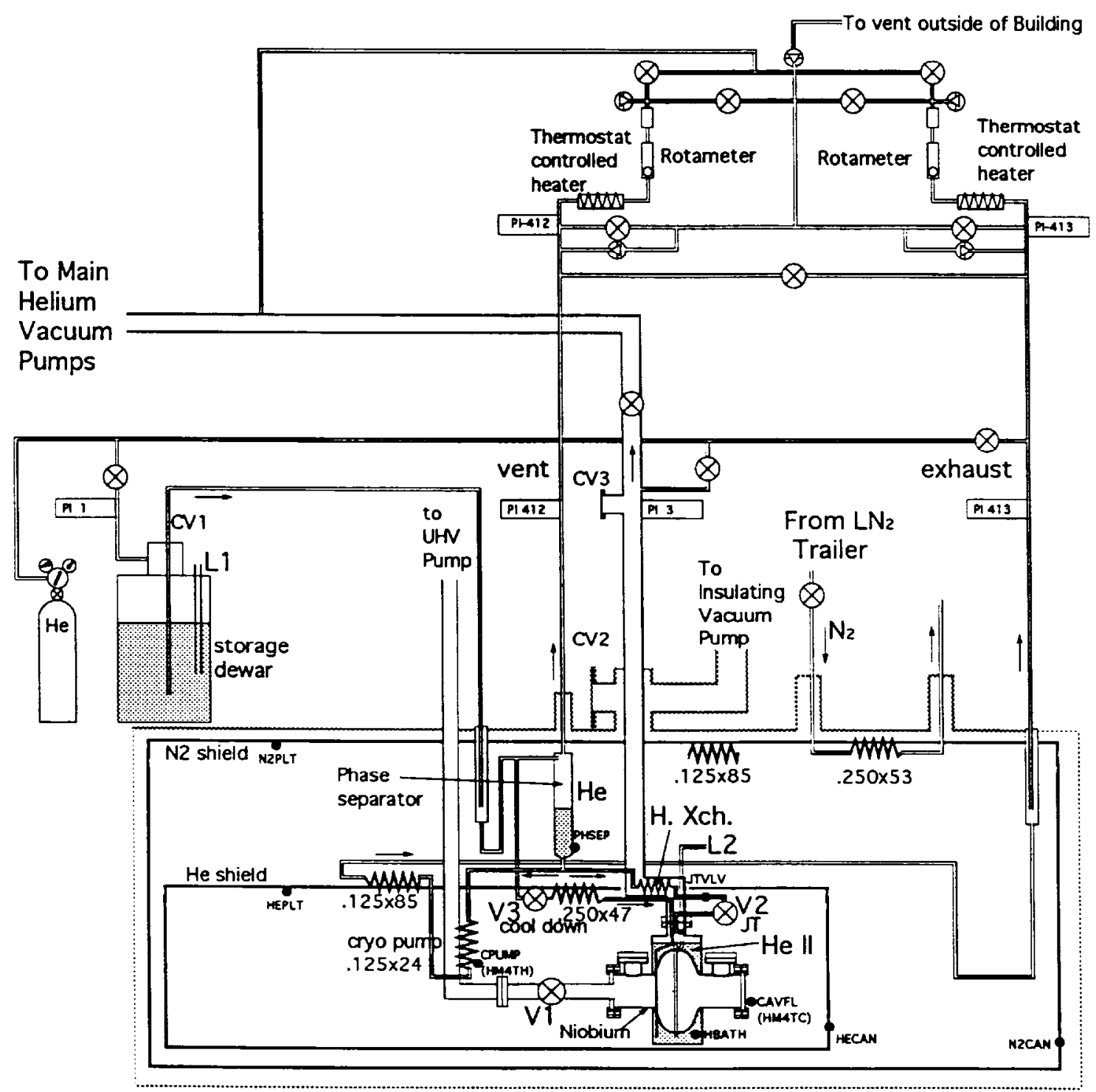

Figure 4. Flow diagram of the cryostat 
Fermilab for control of its Linac ${ }^{6}$. The hardware consists of two modules both of which are nodes in the Internet. One of them is an RF wave form digitizer and the other is the Internet Rack Monitor (IRM) which has been used here for the cryogenic data acquisition. A special set of instrumentation amplifiers ${ }^{7}$ and IRM software ${ }^{8}$ was developed and implemented to handle RTD thermometry. The Parampage ${ }^{9}$ software used is specifically written for MacIntosh computers and communicates with the rest of the hardware by local network or the Internet. So data acquisition (and control if properly authorized) can be monitored independently and simultaneously from anywhere. Most of the time, trouble shooting and resetting operations have been done by the specialists in the Linac Control group at their desks a few kilometers away.

\section{RESULTS}

Besides a commissioning run without power couplers, only one attempt has been made to run this cryostat in the proposed resonant ring mode but the low $Q$ due to the yet unpolished cavity surface prevented resonance. So the data acquired so far are the heat loads of the first two power couplers (carrying no RF power) and the operational parameters for the cryostat itself.

These heat loads include contribution of the infrared radiation exchange between the braided copper connection and its surroundings. They are a function of the temperature distribution throughout the cryostat and are shown in Table 1 . We estimate the order of the uncertainty here to be less than $30 \%$. More running experience and tests are needed to reduce this estimate uncertainty. At this time we are not in need of a more precise result.

Table 1. Power Coupler heat loads

\begin{tabular}{lcccc}
\hline & window load & at & base load & at \\
\hline Coupler \#l & $6.7 \mathrm{~W}$ & $102 \mathrm{~K}$ & $1.01 \mathrm{~W}$ & $6.7 \mathrm{~K}$ \\
Coupler \#2 & $5.8 \mathrm{~W}$ & $101 \mathrm{~K}$ & $0.96 \mathrm{~W}$ & $6.8 \mathrm{~K}$ \\
& & & & \\
\hline
\end{tabular}

Measurements were also made of the temperature of the He bath surrounding the cavity as a function of the power deposited in its internal heater. The steady state temperature was $1.43 \mathrm{~K}$ and Table 2 presents its value as a function of power. The level of the superfluid in the bath went down under the $2 \mathrm{~W}$ and $4 \mathrm{~W}$ loads but it took of the order of 15 minutes to empty.

Table 2. Cavity He Bath temperature under several power loads

\begin{tabular}{lllllllll}
\hline Watt & 0.00 & .129 & .254 & .380 & .498 & 1.00 & 2.00 & 4.01 \\
$\mathrm{~K}$ & 1.43 & 1.45 & 1.48 & 1.50 & 1.52 & 1.60 & 1.71 & 1.86 \\
\hline
\end{tabular}

The operation of the cryostat is best when it is not left to warm up much overnight. This involves an overnight liquid helium consumption rate of 4 liters/hour but it prevents contamination problems and long cooldown times. Under typical measuring conditions, the consumption rate is 32 liters/hour. One feature of the system worth mentioning is how this rate is measured: The American Magnetics superconducting wire level sensor used here is part of an adapter to the commercial $500 \mathrm{~L}$ liquid He container. Its analog voltage output seen as a function of time and the zooming capabilities in the Parampage graphical display provide great sensitivity to the level of the Liquid He supply $( \pm 0.1 \mathrm{~mm})$. This sensitivity allows for the observation of many factors affecting the level. The most obvious the consumption rate reflected in the slope.

\section{CONCLUSION}

A special cryostat has been built and tested at Fermilab capable of measuring the heat 
loads of TESLA input power couplers. The relevance of these measurements is related with the number of these couplers required. The test facilty now under construction in Hamburg requires 32 and the projected accelerator over 20,000 .

\section{ACKNOWLEDGMENTS}

We thank the Cornell U. Laboratory for Nuclear Studies for providing the niobium cavity. A large number of people from Fermilab contributed to this project. In particular we want thank Helen Edwards and Frank Turkot for general support. The following contributions are acknowledged: design and drafting by Clare Miller; machining and welding by Fermilab village machine shop; coordination of assembly and operation by $\mathrm{Al}$ Rusy; level capacitive sensor, heatmeters wiring, cryostat wiring and superinsulation by Dan Assell; level probe electronics by Dyrrell Lewis; heat meter drafting by Clark Reid; general support by Fermilab Lab 2 personnel; data acquisition system setting up and maintenance by Mike Kucera and Bob Florian.

\section{REFERENCES}

1. H. T. Edwards, TESLA Parameters Update - A Progress Report on the TESLA Collider Design, in: "6th Workshop on RF Superconductivity," R.M. Sundelin, ed., CEBAF, Newport News, VA (1993), vol. 1, p. 361.

2. M. Kuchnir, J. D. Gonczy, J. L. Tague, Measuring heat leak with a heatmeter, in: "Advances in Cryogenic Engineering," vol. 31, Plenum Press, New York, (1986), p. 1285.

3. J. Graber, et al., High peak power RF processing studies of $3 \mathrm{GHz}$ niobium cavities, in: "5th Workshop on RF Superconductivity," D. Proch, ed., DESY, Hamburg (1991), vol. 2, p. 758.

4. M. Kuchnir, Fabrication of cryogenic electrical feedthroughs, Fermilab TM-596 (1975).

5. S. J. Collocott, Cryogenics, 327 (1983).

6. R. W. Goodwin, M. J. Kucera, M. F. Shea, Nucl. Instr. and Meth. in Phys. Res. A 352, 189 (1994).

7. R.D. Oberholtzer and B.M. Wisner - private communication

8. R.W. Goodwin - private communication

9. R. E. Peters - private communication 